LA and therefore may be a sensitive biomarker in heralding cardiovascular disease.

This study is the first to examine the association between traditional cardiovascular risk factors and LA structure and function in a large population.

Methods LA volumes were calculated from four- and twochamber CMR cines performed as part of the UK Biobank imaging substudy using the biplane method. To determine phasic function, the LA was automatically contoured in the fourchamber views in all fifty frames of the cardiac cycle using a three-dimensional sparse active shape model (SPASM) with parameters estimated using a deep neural network. A time-volume curve across the cardiac cycle was plotted with a smooth line of best fit (figure 1B). Quality control was performed manually on an initial subset of cases, following which an automated tool was implemented. Utilising the presence of local maxima and minima, LA maximal volume (LAmax), LA minimal (LAmin) volume and LA pre-atrial contraction (LApre) were determined, allowing derivation of total emptying fraction, conduit function and booster function.

Cardiovascular risk factors considered were: age, sex, ethnicity, height, BMI, hypertension, diabetes, hyperlipidaemia, physical activity (metabolic equivalent [MET] minutes/week) and smoking. Multivariable linear regression models were fitted, adjusting for all risk factors with LA parameters as dependent variables.

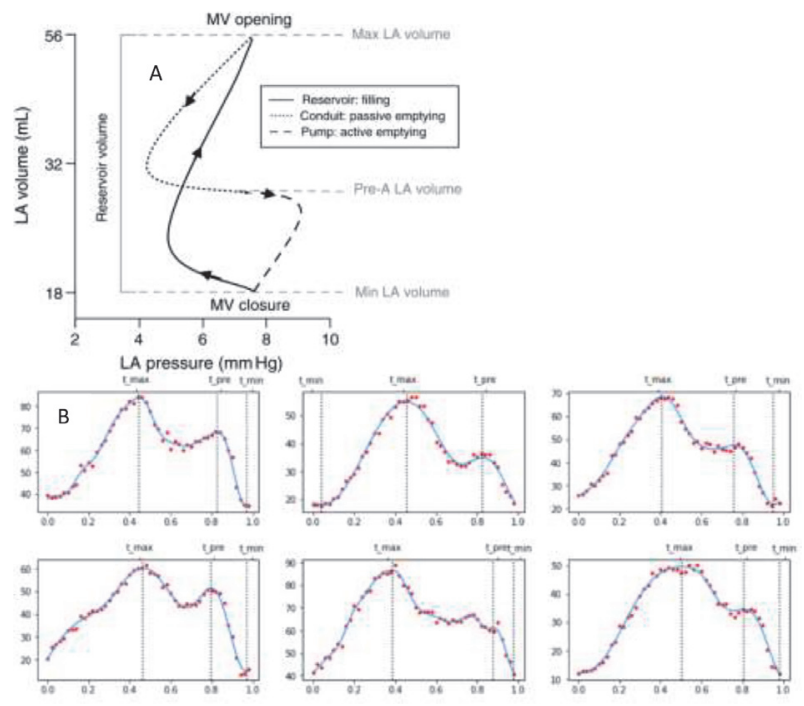

Abstract 13 Figure 1 (A) schematic of atrial phasic function during cardiac cycle. (B) Exemplars of time-volume curves from which atrial function can be derived. $x$-axis represents LA volume in $\mathrm{ml}, \mathrm{y}$-axis is cardiac cycle normalised between 0 and 1 . Functional parameters are derived as follows:

Emptying fraction $=\left(\right.$ LA_max $\left.-L A \_m i n\right) / L A \_m a x$

Conduit function $=\left(\right.$ LA_max $\left.-L A \_p r e\right) / L A \_m a x$

Booster pump $=($ LA_pre - LA_min)/LA_pre

LA size

BMI, HTN, Age = large LA min

BMI, HTN, Age, PA = larger LA max

Diabetes $=$ smaller LA max

Emptying volume and total emptying fraction

BMI, HTN, age = higher emptying volume

$\mathrm{BMI}, \mathrm{HTN}$, age, PA = lower emptying fraction

Conduit function

BMI, HTN, raised chol, smoking $=$ lower conduit function

Booster function

BMI, HTN, age,
Abstract 13 Table 1 Association between cardiovascular risk factors and LA structural and functional parameters

\begin{tabular}{llll}
\hline & Beta & $\begin{array}{l}\text { 95\% confidence } \\
\text { interval }\end{array}$ & p-value \\
\hline LA max (ml) & & & \\
Hypertension & 2.4 & $2.0,2.8$ & $2.3 \times 10^{-33}$ \\
BMI & 1.3 & $1.1,1.4$ & $1.07 \times 10^{-42}$ \\
Diabetes & -1.5 & $-2.2,0.7$ & 0.0001 \\
Total emptying & & & \\
fraction & & & $1.47 \times 10^{-89}$ \\
Hypertension & -0.009 & $-0.01,-0.007$ & $2.77 \times 10^{-18}$ \\
BMI & -0.009 & $-0.01,-0.008$ & $1.15 \times 10^{-136}$ \\
Conduit function & & & $3.85 \times 10^{-05}$ \\
Hypertension & -0.004 & $-0.005,-0.002$ & $9.54 \times 10^{-07}$ \\
BMI & -0.01 & $-0.011,0.009$ & \\
Cigarette smoking & -0.01 & $-0.014,-0.006$ & $1.20 \times 10^{-14}$ \\
Booster pump function & & & $8.89 \times 10^{-31}$ \\
Hypertension & 0.009 & $-0.011,-0.007$ & 0.003 \\
BMI & -0.006 & $-0.007,-0.005$ & \\
Cigarette smoking & -0.003 & $-0.005,-0.001$ & \\
\hline
\end{tabular}

Results After quality control, 44,957 participants were included in the analysis. The mean age was $64 \pm 8,51.8 \%$ of the cohort were female. Regression analysis is detailed in table 1. Hypertension and increasing BMI were significantly associated with lower total emptying fraction, conduit function and booster pump function. Cigarette smoking also resulted in reduced conduit function and booster pump function. Hypertension and BMI were associated with larger LAmax volumes.

Beta coefficients, 95\% confidence intervals and p-values estimating association of risk factors with LA parameters in multivariable linear regression models. Co-variates included in models are as follows: age, sex, ethnicity, height, BMI, hypertension, diabetes, hyperlipidaemia, total MET minutes/week, cigarette smoking.

Conclusion This is the first study to calculate time-volume curves to derive markers of atrial function in a large cohort. We demonstrate that hypertension and increasing BMI - risk factors that are heavily implicated in diastolic dysfunction are most frequently associated with alterations in LA phasic function.

\section{THE PRESENCE OF DIABETES AS A COMORBIDITY ADVERSELY AFFECTS THE PHENOTYPIC EXPRESSION OF HYPERTROPHIC CARDIOMYOPATHY}

${ }^{1}$ Nicholas Jex, ${ }^{1}$ Amrit Chowdhary, ${ }^{1}$ Sharmaine Thirunavukarasu, ${ }^{2}$ Henry Procter, ${ }^{2}$ Anshuman Sengupta, ${ }^{1}$ Pavithra Natarajan, ${ }^{2}$ Ana-Maria Poenar, ${ }^{1}$ Peter Swoboda, ${ }^{3}$ Hui Xue, ${ }^{1}$ Richard M Cubbon, ${ }^{3}$ Peter Kellman, ${ }^{1}$ John P Greenwood, 'Sven Plein, ${ }^{2}$ Stephen Page, ${ }^{1}$ Eylem Levelt. 'University of Leeds, Multidisciplinary Cardiovascular Research Centre and Biomedical Imaging Science Department, Leeds Institute of Cardiovascular and Metabolic Medicine, LS2 9JT, UK; ${ }^{2}$ Leeds Teaching Hospitals NHS Trust, Department of Cardiology, Leeds, LS1 3EX, UK; ${ }^{3}$ National Heart, Lung, and Blood Institute, National Institutes of Health, DHHS, 10 Center Drive MSC-1061, Bethesda, MD, 20892, USA

\subsection{6/heartjnl-2021-BSCMR.14}

Background Type 2 diabetes mellitus (DM) is associated with worsened clinical outcomes in hypertrophic cardiomyopathy (HCM) patients, although the mechanisms of this association are incompletely understood. 
Objectives Utilizing ${ }^{31}$ Phosphorus magnetic resonance spectroscopy ( $\left.{ }^{31} \mathrm{P}-\mathrm{MRS}\right)$ and cardiovascular magnetic resonance (CMR) in a prospective observational study of groups of patients with concomitant HCM and DM (HCM-DM), isolated HCM, isolated DM and healthy volunteers (HV), we tested the hypotheses that the collective impact of HCM and $\mathrm{DM}$ on the myocardium aggravates myocardial energetic impairment, contractile dysfunction, and fibrosis, and impairs coronary microvascular function.

Methods Seventy-five age- and sex-matched participants were recruited (20 HCM-DM, 20 HCM, 20 DM and $15 \mathrm{HV}$ ). HCM groups were matched for HCM phenotype. DM groups were matched for diabetes treatment, duration and HbA1c.

\section{Abstract 14 Table 1 CMR and ${ }^{31}$ P-MRS findings}

\begin{tabular}{|c|c|c|c|c|c|}
\hline & HV $n=15$ & DM $n=20$ & HCM $n=18$ & HCM-DM n=20 & $P$ value \\
\hline $\mathrm{LV}$ end-diastolic volume indexed to $\mathrm{BSA}, \mathrm{mL} / \mathrm{m}^{2}$ & $83 \pm 18$ & $75 \pm 16$ & $82 \pm 19$ & $76 \pm 22$ & 0.09 \\
\hline LV end-systolic volume indexed to BSA, $\mathrm{ml} / \mathrm{m}^{2}$ & $31 \pm 79$ & $32 \pm 10 \S$ & $28 \pm 15$ & $26 \pm 14$ & 0.001 \\
\hline LV mass, $g$ & $99 \pm 279 \dagger$ & $83 \pm 23^{\star} \S$ & $173 \pm 63$ & $187 \pm 73$ & $<0.0001$ \\
\hline LV mass index, $\mathrm{g} / \mathrm{m}^{2}$ & $54 \pm 119 \dagger$ & $44 \pm 12 \S$ & $90 \pm 27^{*}$ & $92 \pm 40$ & $<0.0001$ \\
\hline LV mass to LV end-diastolic volume, $\mathrm{g} / \mathrm{mL}$ & $0.66 \pm 0.119$ & $0.61 \pm 0.15 \S$ & $1.03 \pm 0.31^{*}$ & $1.24 \pm 0.36$ & $<0.0001$ \\
\hline LV stroke volume, ml & $95 \pm 239$ & $80 \pm 17 \S$ & $118 \pm 21^{*}$ & $101 \pm 22$ & $<0.0001$ \\
\hline LV ejection fraction, $\%$ & $63 \pm 4 \dagger$ & $58 \pm 7 \S$ & $70 \pm 9^{*}$ & $67 \pm 9$ & $<0.0001$ \\
\hline LV maximal wall thickness, mm & $10 \pm 19 \dagger$ & $9 \pm 2 * \S$ & $20 \pm 2$ & $21 \pm 4$ & $<0.0001$ \\
\hline $\mathrm{RV}$ end-diastolic volume indexed to $\mathrm{BSA}, \mathrm{mL} / \mathrm{m}^{2}$ & $86 \pm 209$ & $69 \pm 13 \neq$ & $79 \pm 14$ & $66 \pm 13$ & 0.0006 \\
\hline $\mathrm{RV}$ end-systolic volume indexed to $\mathrm{BSA}, \mathrm{ml} / \mathrm{m}^{2}$ & $35 \pm 10$ & $30 \pm 10$ & $30 \pm 10$ & $28 \pm 13$ & 0.35 \\
\hline RV stroke volume, ml & $95 \pm 239$ & $73 \pm 16 \neq^{*}$ & $94 \pm 16 €$ & $75 \pm 21$ & 0.0006 \\
\hline RV ejection fraction, $\%$ & $60 \pm 6$ & $58 \pm 8$ & $62 \pm 8$ & $58 \pm 13$ & 0.44 \\
\hline LA biplane end-systolic volumes, $\mathrm{mL}$ & $67 \pm 179 \dagger$ & $62 \pm 18 \S$ & $100 \pm 28^{*}$ & $113 \pm 59$ & $<0.0001$ \\
\hline Biplane LA EF,\% & $62 \pm 79 \dagger$ & $54 \pm 10 \S$ & $45 \pm 10 €$ & $34 \pm 18$ & $<0.0001$ \\
\hline Global longitudinal strain, negative $(-), \%$ & $14 \pm 39$ & $15 \pm 4 \S$ & $13 \pm 3 €$ & $10 \pm 4$ & 0.0007 \\
\hline Peak systolic circumferential strain, $(-), \%$ & $21 \pm 29$ & $18 \pm 6$ & $20 \pm 4 €$ & $16 \pm 4$ & 0.009 \\
\hline Peak circumferential diastolic strain rate, $\mathrm{s}^{-1}$ & $1.19 \pm 0.24 \uparrow$ & $1.05 \pm 0.32 \S$ & $0.99 \pm 0.21 €$ & $0.87 \pm 0.22$ & 0.006 \\
\hline Mean native $\mathrm{T} 1$, (ms) & $1211 \pm 81$ & $1205 \pm 107$ & $1211 \pm 65$ & $1209 \pm 69$ & 0.9 \\
\hline Extra cellular volume, (\%) & $25 \pm 29$ & $23 \pm 3 \S$ & $28 \pm 7^{*}$ & $36 \pm 10$ & $<0.0001$ \\
\hline LGE scar percentage of LV mass (\%) & & & $4 \pm 4$ & $10 \pm 8$ & 0.002 \\
\hline \multicolumn{6}{|c|}{ Myocardial Energetics } \\
\hline PCr/ATP ratio & $2.17 \pm 0.499$ & $1.58 \pm 0.21 \neq$ & $1.93 \pm 0.38 €$ & $1.54 \pm 0.27$ & 0.0002 \\
\hline \multicolumn{6}{|c|}{ Myocardial Perfusion } \\
\hline Increase in RPP,\% & 37 & 39 & 33 & 32 & 0.3 \\
\hline Stress $\mathrm{MBF}, \mathrm{ml} / \mathrm{min} / \mathrm{g}$ & $2.06 \pm 0.42 ף$ & $1.95 \pm 0.41 \S$ & $1.74 \pm 0.44 €$ & $1.39 \pm 0.42$ & 0.0017 \\
\hline Rest MBF, $\mathrm{ml} / \mathrm{min} / \mathrm{g}$ & $0.68 \pm 0.03$ & $0.72 \pm 0.17$ & $0.59 \pm 0.19$ & $0.69 \pm 0.16$ & 0.05 \\
\hline MPR & $3.19 \pm 0.79$ 凤 & $2.75 \pm 0.64 \S$ & $3.09 \pm 1.06 €$ & $2.04 \pm 0.82$ & 0.0012 \\
\hline
\end{tabular}

$€$ signifies $p<0.05$ between HCM-DM and HCM, $\S$ signifies $p<0.05$ between HCM-DM and DM.

I signifies $p<0.05$ between HCM-DM and HV * signifies $p<0.05$ between HCM and DM.

$\dagger$ signifies $p \leq 0.05$ between HCM and HV, $\neq$ signifies $p \leq 0.05$ between DM and HV.

Values are mean \pm standard deviations or percentages. BSA indicates body surface area; LV, Left ventricle; RV, right ventricle; DM, type 2 diabetes mellitus; HCM, hypertrophic cardiomyopathy; LV, left ventricular; LA, left atrial; LA EF, left atrial ejection fraction; LGE, late gadolinium enhancement; PCr, phosphocreatine; ATP, adenosine tri-phosphate; RPP, rate pressure product; MBF, myocardial blood flow; MPR, myocardial perfusion reserve.
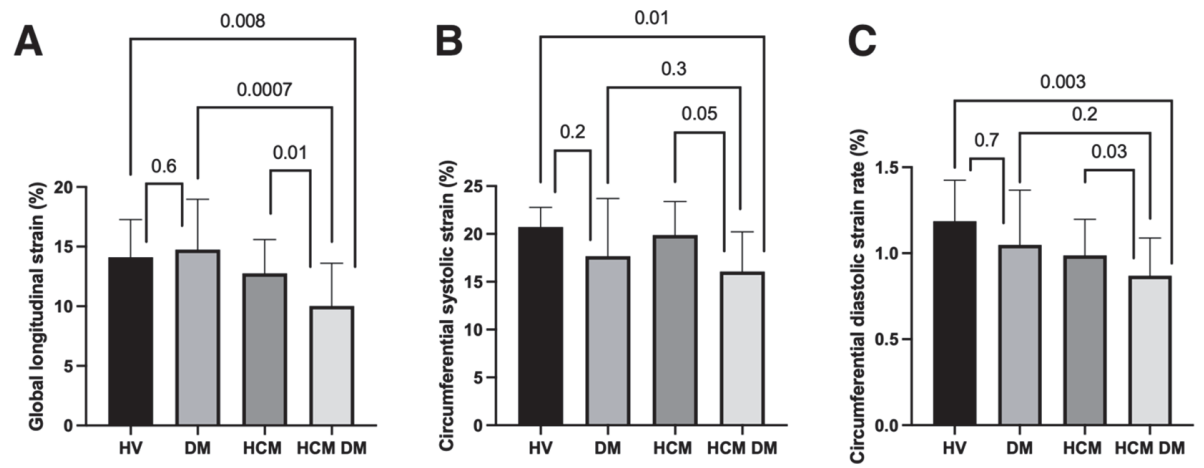

Abstract 14 Figure 1 Differences in global longitudinal strain, circumferential systolic strain and peak diastolic strain rate parameters between healthy volunteers, patients with isolated DM, patients with isolated HCM and patients with HCM and DM; (A) Global longitudinal strain\%, (B) Circumferential systolic strain $\%$, (C) peak circumferential diastolic strain rate $\mathrm{s}^{-1}$. 

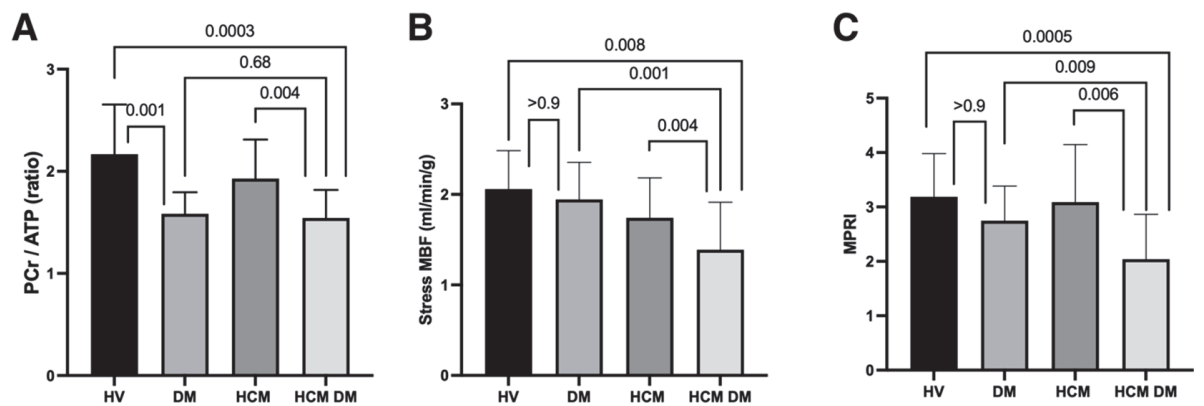

Abstract 14 Figure 2 Differences in myocardial PCr/ATP ratio, global stress myocardial blood flow and myocardial perfusion reserve between healthy volunteers, patients with isolated DM, patients with isolated HCM and patients with HCM and DM; (A) PCr/ATP ratio; (B) Global stress myocardial blood flow (ml/min/g), (C) Myocardial perfusion reserve.
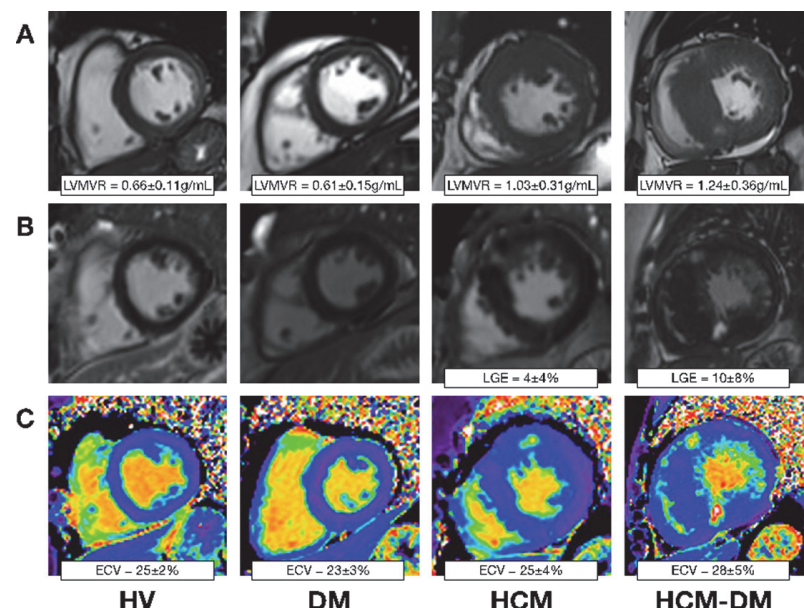

DM

HCM

HCM-DM

Abstract 14 Figure 3 Representative examples of mid-left ventricular short axis cine imaging (row A with group mean values and standard deviations provided for the LV mass over LV end-diastolic volume ratios); late gadolinium enhancement imaging (row B, with group mean values and standard deviations provided for the LGE scar percentage of LV mass for the two HCM groups where scar was present); post-contrast native T1 maps (row C, with group mean values and standard deviations provided for the extracellular matrix volume fractions) from a healthy volunteer (first column), a patient with isolated DM (second column), a patient with isolated HCM (third column) and a patient with HCM and DM (fourth column).

HV
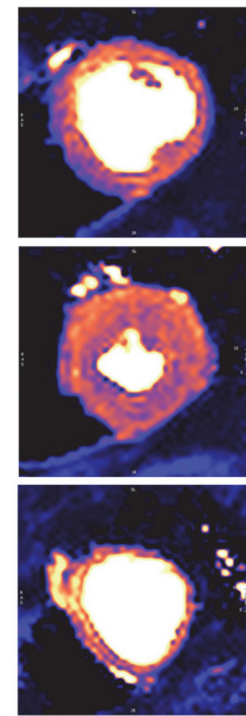

Stress MBF 2.06 $\Rightarrow \mathrm{ml} / \mathrm{min} / \mathrm{g}$
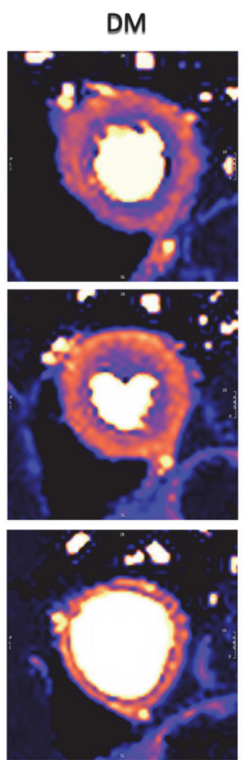

Stress MBF 1.95 $\mathrm{ml} / \mathrm{min} / \mathrm{g}$
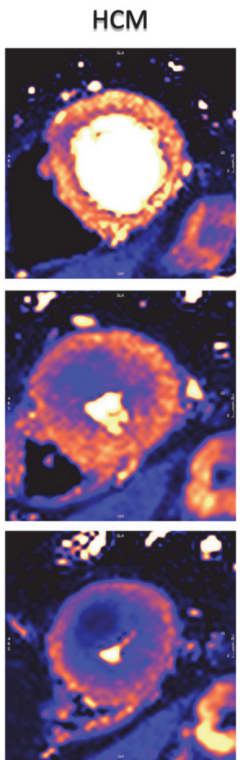

Stress MBF 1.74 $\mathrm{ml} / \mathrm{min} / \mathrm{g}$
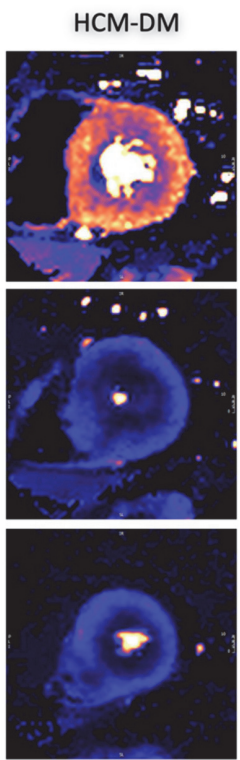

0.0

Stress MBF 1.39 $\mathrm{ml} / \mathrm{min} / \mathrm{g}$

Abstract 14 Figure 4 Representative examples of mid-left ventricular stress perfusion maps from a healthy volunteer (first column), a patient with isolated DM (second column), a patient with isolated HCM (third column) and a patient with HCM and DM (fourth column). 
Results ESC sudden cardiac death risk scores were comparable between the HCM groups (HCM:2.2 $\pm 1.5 \%$, HCM-DM:1.9 $\pm 1.2 \% ; \mathrm{p}=\mathrm{NS})$ and sarcomeric mutations were equally common. HCM-DM had the highest NT-proBNP levels (HV:42 ng/L[IQR:35-66 ng/L], DM:118 ng/L[IQR:53-187 ng/L], HCM:298 ng/L[IQR:157-837 ng/L], HCM-DM:726 ng/L [IQR:213-8695 ng/L]; $\mathrm{p}<0.0001)$. Left-ventricular ejection fraction, mass and wall thickness were similar between the HCM groups. HCM-DM displayed a greater degree of fibrosis burden with higher extracellular volume fraction and scar percentage, more significant reductions in global longitudinal strain and left atrial function compared to the isolated HCM. PCr/ATP was similarly decreased in the HCM-DM and DM (HV:2.17 \pm 0.49 , DM:1.58 \pm 0.27, HCM:1.93 \pm 0.38 , HCMDM:1.54 $\pm 0.27 ; \mathrm{p}=0.0002)$. HCM-DM had the lowest stress myocardial blood flow (HV:2.06 $\pm 0.42 \mathrm{ml} / \mathrm{min} / \mathrm{g}$, DM:1.95 $\pm 0.41 \mathrm{ml} / \mathrm{min} / \mathrm{g}$, HCM:1.74 $\pm 0.44 \mathrm{ml} / \mathrm{min} / \mathrm{g}$, HCM-DM:1.39 $\pm 0.42 \mathrm{ml} / \mathrm{min} / \mathrm{g} ; \mathrm{p}=0.0017$ ).

Conclusions Comorbid diabetes adversely affects the HCM phenotype with greater reductions in myocardial energetics, perfusion, strain, increased scar burden and higher NT-proBNP levels compared to isolated HCM. Our findings suggest that specific, targeted therapeutic approaches may be useful in hypertrophic cardiomyopathy patients with diabetes comorbidity to improve clinical outcomes.

\section{THE ROLE OF CARDIAC MRI IN ASSESSING BICUSPID AORTIC VALVE DISEASE AND ASSOCIATED MYOCARDIAL FIBROSIS}

Nikhil Chatrath, Raghav Bhatia, Saad Fyyaz, Hamish Maclachlan, Sanjay Sharma, Maite Tome, Michael Papadakis. St George's University, London

\subsection{6/heartjnl-2021-BSCMR.15}

Introduction Bicuspid aortic valve (BAV) is the most common congenital cardiovascular abnormality. Cardiac MRI (CMR) is commonly used to screen for aortic dilatation and associated abnormalities. Sudden death has been reported, often due to aortic stenosis (AS) or dissection, but it has been hypothesised that areas of fibrosis may be a substrate for arrythmias in patients with BAV.

Methods The aim of this study was to review the CMR findings of consecutive patients with BAV including patterns of late gadolinium enhancement (LGE). Valve morphology was described according to recent international guidance ${ }^{1}$. The prevalence of aortic dilatation, left ventricular hypertrophy $(\mathrm{LVH})$, AS, aortic regurgitation (AR) and the distribution of late gadolinium enhancement (LGE) was noted.

Results 217 patients with BAV had CMR performed at a single-centre between May 2011 and September 2021; mean age 46 years (IQR 32-62), 74.6\% male $(n=162)$. 6.5\% $(n=14)$ had an aortic valve replacement (AVR). 25.8\% $(n=56)$ had at least moderate AS and $17.5 \%(n=38)$ at least moderate AR, quantified by flow measurements.

Valve morphology could be established in 161 cases; $89.4 \%$ $(n=144)$ demonstrated a fused BAV, the majority demonstrating right-left cusp fusion $(73 \% ; n=105) .164$ had a full aortogram performed, of which $9.8 \% \quad(n=16)$ had evidence of aortic coarctation. When indexed to body surface area, $28.7 \%$ $(n=47)$ had dilatation at the sinus of Valsalva, $39.0 \%$ $(n=64 \%)$ at the sino-tubular junction and 56.2\% $(n=94)$ at the ascending aorta.
Delayed enhancement images were acquired in 116 studies and LGE was identified in $20.7 \%(n=24)$ of these. In $45.8 \%$ $(n=11)$, the LGE could be attributed to other causes, most commonly infarction, with significant coronary artery disease confirmed on angiography $(n=9)$. In the remaining subjects, none of whom had evidence of AS or LVH, there was midwall LGE in 13 subjects; predominantly in the basal septum $(n=7)$, lateral wall $(n=3)$, inferior RV insertion point $(n=2)$ and anterior wall $(n=1)$.

Conclusion This study highlights the multiple roles of CMR in the assessment of individuals with BAV. Further larger-scale studies are required to analyse patterns of LGE to try and better understand the distribution of fibrosis in these patients, and to correlate these findings with clinical outcomes.

\section{REFERENCE}

1. Michelena HI, Della Corte A, Evangelista A, et al. International consensus statement on nomenclature and classification of the congenital bicuspid aortic valve and its aortopathy, for clinical, surgical, interventional and research purposes. J Thorac Cardiovasc Surg. 2021;162(3):e383-e414. doi:10.1016/j. jtcvs.2021.06.019

\section{VALIDATION OF TIME-RESOLVED, AUTOMATED PEAK TRANSVALVULAR VELOCITY TRACKING THROUGH THE MITRAL VALVE USING FOUR-DIMENSIONAL FLOW CARDIOVASCULAR MAGNETIC RESONANCE}

\begin{abstract}
${ }^{1,2}$ Paul Njoku, ${ }^{2}$ Ciaran Grafton-Clarke, ${ }^{1}$ Hosam Assadi, ${ }^{3}$ Rebecca Gosling, ${ }^{3}$ Gareth Archer ${ }^{1,2}$ Marcus Flather, ${ }^{1,2}$ Vassilios Vassiliou, ${ }^{1,2}$ Pankaj Garg. ${ }^{1}$ University of East Anglia, Norwich Medical School, Norfolk, UK; ${ }^{2}$ Norfolk and Norwich University Hospitals NHS Foundation Trust, Norfolk, UK; ${ }^{3}$ University of Sheffield Medical School and Sheffield Teaching Hospitals NHS Trust, Sheffield, UK
\end{abstract}

\subsection{6/heartjnl-2021-BSCMR.16}

Doppler echocardiography (TTE) remains the imaging modality of choice for the assessment of mitral inflow and left ventricular diastolic function, despite its limitations. Four-dimensional flow cardiovascular magnetic resonance (4D flow CMR) offers time-resolved cross-sectional velocity data, which can be used to investigate transvalvular peak velocity through the mitral valve. This would not suffer from the in-plane motion and angle-dependence of pulsewave echocardiography.

Objective We aim to validate a novel time-resolved, automated dynamic 4D flow CMR peak velocity tracking method for measuring the peak velocity of mitral inflow against TTE.

Method Patients recruited to EurValve programme $(n=22)$ underwent TTE and 4D flow CMR. Peak E-wave and A-wave velocities were recorded. This work was done in collaboration with the industry leader in 4D flow CMR (PIE Medical Imaging). Transvalvular flow segmentation was done using established valve tracking methods and the generated $3 \mathrm{D}$ streamlines were investigated for seeking the peak velocity inside the left ventricular cavity during diastole. Reproducibility analyses were carried out in 10 cases.

Results The peak E-wave mitral inflow velocity was comparable between the novel 4D flow method and TTE $(1.09 \pm$ $0.29 \mathrm{~m} / \mathrm{s}$ and $1.10 \pm 0.37 \mathrm{~m} / \mathrm{s}$ respectively; $\mathrm{p}=0.60)$. The mean A-wave peak velocity was also comparable across both methods $(0.94 \pm 0.40 \mathrm{~m} / \mathrm{s}$ and $0.86 \pm 0.29 \mathrm{~m} / \mathrm{s}$ respectively; $\mathrm{p}=0.38$ ). The automated $4 \mathrm{D}$ flow method also showed good correlation with TTE for both E-wave $(\mathrm{r}=0.54 ; \mathrm{p}=0.01)$ and A-wave $(\mathrm{r}=0.55 ; \mathrm{p}=0.03)$ with 